\title{
Volume Free Electron Laser with a "Grid" Photonic Crystal in a Cylindrical Waveguide
}

\author{
V.G. BarysheVsKY* AND P.V. MOlChanov \\ Research Institute for Nuclear Problems, 11 Bobryiskaya str., 220030, Minsk, Belarus
}

\begin{abstract}
Specific features of Volume Free Electron Laser (VFEL) with a photonic crystal made from the wire grids periodically strained in a waveguide are studied theoretically and experimentally. Electrodynamical properties of a "grid" photonic crystal built in a cylindrical waveguide are reported. Possibility to use it for VFEL lasing in wide frequency range is discussed.
\end{abstract}

PACS numbers: 41.60.Cr, 41.75.Fr, 41.75.Ht, 42.79.Dj

\section{Introduction}

Traveling Wave Tube (TWT)-like and Backward Wave Oscillators (BWO)-like generators are now widespread $[1,2]$. New type of TWT and BWO on the basis of Volume Free Electron Laser (VFEL) [3-5] was proposed in [6-9]. Transverse dimensions of VFEL resonator could significantly exceed radiation wavelength. The electron beam and radiation power are distributed over the large volume that is beneficial for electrical endurance of the system. One of the VFEL types uses a "grid" photonic crystal that is formed by a periodically strained threads either dielectric or metallic. Theoretical analysis $[6,7]$ showed that the "grid" photonic crystal, made of metal threads, is almost transparent for the electromagnetic waves in the frequency range from $\mathrm{GHz}$ to $\mathrm{THz}$. In the present paper different types of "grid" photonic crystals are analyzed for VFEL operation. Wave dispersion in a "grid" photonic crystal built in a circular waveguide is considered.

\section{Experimental and numerical studies of "grid" photonic crystals}

In our first experiments [8] we used the photonic crystal built inside rectangular cross-section waveguide. Rows of tungsten threads were strained inside the rectangular waveguide (see Fig. 1). A pencil-like electron beam

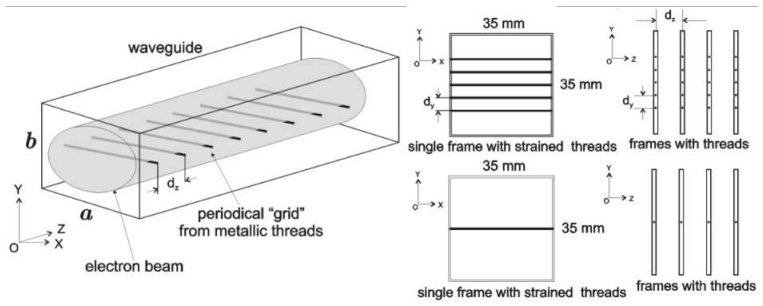

* corresponding author; e-mail: bar@inp.minsk.by
Fig. 1. The "grid" diffraction grating placed inside the waveguide.

with longitudinal energy up to $100 \mathrm{keV}$ passes through the above structure. Period of "grid" photonic crystal is chosen to provide radiation frequency $\sim 8.4 \mathrm{GHz}$. The "grid" structure is made of separate frames each containing the layer of 1, 3 or 5 parallel threads (see Fig. 1). Frames are joined to get the "grid" structure with the distance $d_{z}$ between layers.

Stability of "grid" to heating by electron beam was evaluated and confirmed by experiments with VFEL with the different "grids" $[8,9]$.

In the experiments $[8,9]$ generation of radiation in the "grid" VFEL was observed in BWO regime. The sample oscillogram is shown in Fig. 2a, where signals marked 1 and 2 are the signals obtained from microwave detectors. Other two curves are the electron gun voltage and electron beam current. Time scale is $80 \mathrm{~ns}$.

The radiation power was measured for photonic crystal with 4, 6, 10, 12, 14 and 22 frames (period $12.5 \mathrm{~mm}$ ) each containing five threads. Dependence of the generated radiation intensity on the "grid" photonic crystal length is obtained. The result of these measurements is presented in Fig. 2b. It can be seen from this figure that for the photonic crystal with more than 14 periods $(\sim 3 \lambda)$ saturation is reached.

The next experiment uses "grid" resonator made of two photonic crystals with different periods. Period of the second photonic crystal is chosen to provide for the electron beam, which has lost part of its energy for radiation in the first photonic crystal, the same radiation frequency. Two radiation peaks are apparent in these experiments. Measurements of radiation frequency give value close to $8.4 \mathrm{GHz}$ for both peaks. Their intensities are also comparable and, as a result, the detected output is almost twice higher than that for the experiments with "grid" photonic crystal with fixed period. Experimental results confirmed conclusion that photonic crystal with variable period could increase radiation output.

The main disadvantage of VFEL based on such a type of a "grid" photonic crystal (see Fig. 1) is the pres- 


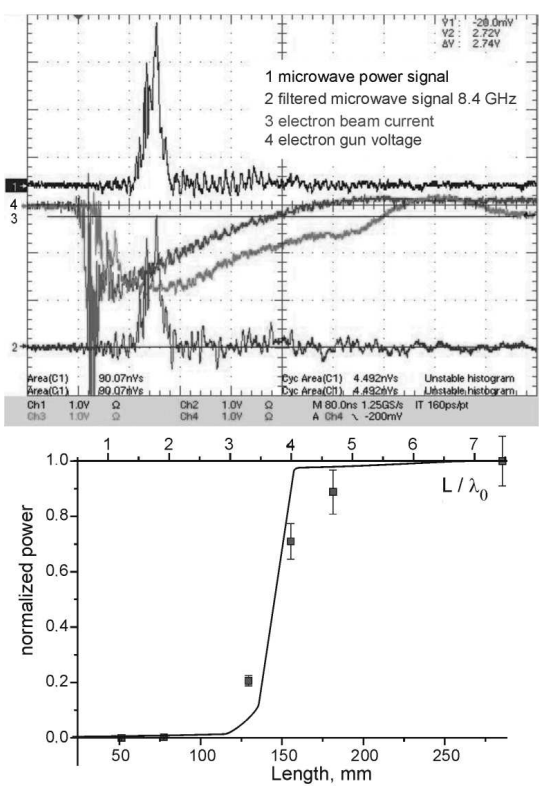

Fig. 2. (a) The sample oscillogram. (b) Dependence of the radiation power on the length of the "grid" photonic crystal.

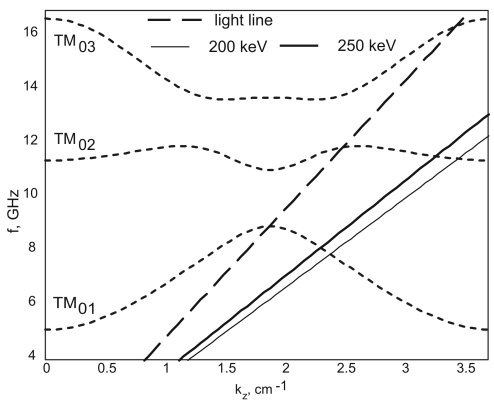

Fig. 3. Dispersion diagrams of $\mathrm{TM}_{01,02,03}$ modes for "grid" photonic crystal built in circular waveguide.

ence of additional perturbations of the electron beam cross-section. While axially-symmetrical electron beam is guided through the rectangular waveguide by an external magnetic field, the presence of waveguide edges causes additional electromagnetic forces. These electromagnetic forces sufficiently influence trajectories of electrons and may bring to the spike oscillation (see Fig. 2a) in radiation process that is extremely important for lasing in millimeter wavelength range. The most effective way to avoid such a problem is application of waveguides with circular cross-section with fine surface treatment.

Like a photonic crystal built inside rectangular waveguide the structure of new type contains separated frames with 1, 3 or 5 parallel threads. These frames form "large" period of the structure, which could comprise up to 7 "small" periods in one row. The number of small periods can be mechanically changed. The developed structure is represented as 3D "grid" photonic crystal.
The dispersion diagrams for the lower operating and the neat upper TM modes are numerically calculated for the "grid" photonic crystal in circular waveguide with internal diameter $D=50 \mathrm{~mm}$ and structure period $d=16 \mathrm{~mm}$ with 5 threads in a period. The results of calculation for the lower TM01 mode are presented in Fig. 3 .

This diagram is similar to that for circular corrugated waveguides, which are conventionally applied for TWT and BWO. Therefore, such a photonic crystal made of metallic threads strained inside the circular waveguide can be used for VFEL lasing in TWT and BWO regimes in our future experiments with the electrons beam energy in range of $200-250 \mathrm{keV}$.

\section{Conclusion}

Theoretical and experimental analysis shows that the cylindrical waveguide with the photonic crystal of metallic threads periodically strained inside it can be used for VFEL lasing in TWT and BWO regimes. Wave dispersion in a "grid" photonic crystal built in a circular waveguide is considered.

\section{Acknowledgments}

The work was supported by the Belarusian Fundamental Science Foundation grant No. $\Phi 07 \mathrm{~B}-001$.

\section{References}

[1] V.L. Granatstein, R.K. Parker, C.M. Armstrong, in Proceedings of the IEEE, 87, 5, 702 (1999).

[2] S.P. Bugaev, V.A. Cherepenin, V.I. Kanavets, A.I. Klimov, A.D. Kopenkin, V.I. Koshelev, V.A. Popov, A.I. Slepkov, IEEE Trans. Plasma Sci. 18, 518 (1990).

[3] V.G. Baryshevsky, NIM A 445, 281 (2000); LANL e-print archive physics/9806039.

[4] V.G. Baryshevsky, K.G. Batrakov, A.A. Gurinovich, J.J. Ilienko, A.S. Lobko, V.I. Moroz, P.F. Sofronov, V.I. Stolyarky, NIM A 483, 21 (2002).

[5] V.G. Baryshevsky, K.G. Batrakov, V. Mihalchik, V.P. Peftiev, V.I. Stolyarsky, Eurasian patent EA 004665B1.

[6] V.G. Baryshevsky, A.A. Gurinovich, LANL e-print archive: physics/0409107.

[7] V.G. Baryshevsky, A.A. Gurinovich, NIM B 252, 92 (2006).

[8] V.G. Baryshevsky, K.G. Batrakov, N.A. Belous, A.A. Gurinovich, A.S. Lobko, P.V. Molchanov, P.F. Sofronov, V.I. Stolyarky, LANL e-print archive: physics/0409125.

[9] V.G. Baryshevsky, N.A. Belous, A.A. Gurinovich, A.S. Lobko, P.V. Molchanov, V.I. Stolyarsky, in Proceedings of FEL, Novosibirsk, Russia 331 (2006), LANL e-print arXiv: physics/0605122. 